\title{
The Bandwidth Exchange Architecture
}

\author{
David Michael Turner \\ Dept. of Computer Science \\ Drexel University \\ dmt36@drexel.edu
}

\author{
Vassilis Prevelakis \\ Dept. of Computer Science \\ Drexel University \\ vp@cs.drexel.edu
}

\author{
Angelos D. Keromytis \\ Dept. of Computer Science \\ Columbia University \\ angelos@cs.columbia.edu
}

\begin{abstract}
New applications for the Internet such as video on demand, grid computing etc. depend on the availability of high bandwidth connections with acceptable Quality of Service $(Q o S)$. There appears to be, therefore, a requirement for a market where bandwidth-related transactions can take place. For this market to be effective, it must be efficient for both the provider (seller) and the user (buyer) of the bandwidth. This implies that: (a) the buyer must have a wide choice of providers that operate in a competitive environment, (b) the seller must be assured that a QoS transaction will be paid by the customer, and (c) the QoS transaction establishment must have low overheads so that it may be used by individual customers without a significant burden to the provider.

In order to satisfy these requirements, we propose a framework that allows customers to purchase bandwidth using an open market where providers advertise links and capacities and customers bid for these services. The model is close to that of a commodities market that offers both advance bookings (futures) and a spot market. We explore the mechanisms that can support such a model.
\end{abstract}

\section{Introduction}

Years of research on Quality of Service (QoS) architectures for the Internet have resulted in sophisticated proposals that have not been broadly exploited commercially. In particular, Integrated Services (IntServ) [4] and Differentiated Services (DiffServ) [1] have long been supported by major router and operating system vendors, yet have only seen minimal use in practice. One explanation offered by the networking and QoS community has been a lack of a commercialization model, together with the necessary accounting and charging architecture [7]. A related crucial issue is assurance of end-to-end QoS coherence in the face of multiple intervening parties, such as transit ISPs.

These two issues, taken together, are responsible for sup- pressing interest from both the ISPs (in commercially exploiting QoS to its full potential) and the users (in taking advantage of such services). Simply put, if an ISP cannot be paid for reserving bandwidth to a user, they will not offer QoS; if users cannot be assured of end-to-end QoS, they will not pay for the service. Compounding the problem is the issue of management: it is certainly possible for a large entity, such as a multi-national company, to coordinate with the relevant ISPs so that its various geographically dispersed networks are connected provisioned using a series of DiffServ or IntServ tunnels. However, the effort is considerable and requires manual intervention from a number of people. Perhaps most importantly, the ISPs' network operations centers (NOCs) will need to configure the various routers appropriately. Clearly, such an approach will not scale well if preferentially treated bandwidth is to become a commodity that can be traded, as has been recognized before [5]. Yet, the increasing use of the Internet for time-sensitive or otherwise critical applications effectively mandate some form of bandwidth reservation, often for short periods of time (e.g., watching a movie).

We present a market-based approach to self-managing QoS across multiple ISPs. Our architecture introduces a Bandwidth Exchange (BAND-X), which facilitates the trading of reserved bandwidth between ISPs and users. This facility allows purchasing bandwidth in advance (effectively creating a "futures" market for bandwidth) as well as on the "spot" market. Users can select from a range of offerings by various ISPs to create an end-to-end pipe (with the desired bandwidth and QoS) piece-meal, or can choose to purchase a complete package from a single provider (or consortium of providers), where available. This is similar to the way people purchase low-cost airplane tickets online.

To ease the task of accounting and administration, we use the micropayment architecture introduced in [3] to provide both accounting and authorization. Briefly, users purchasing bandwidth on BAND- $X$ are provided with credentials that allow them to establish the necessary QoS pipes among the necessary network elements (routers), within the constraints of their contracts. Our use of a trust-management 
system (KeyNote [2]) allows us to perform both billing and authorization with the same mechanism, simplifying the architecture and eliminating the need for manual configuration or universal trust of the BAND-X service (e.g., to configure the relevant routers of several ISPs).

To better illustrate the use of the BAND-X architecture, we next describe a sample usage scenario involving an end user and several ISPs. In Section 2 we present the system architecture in more detail. Section 3 describes the various components of our system, in particular our micro-checks mechanism, and how they operate together, along with a security analysis.

\subsection{Motivation}

Consider the following scenario of a user Alice wishing to reserve an end-to-end 50Mbps "pipe" from Rome to Dublin ${ }^{1}$. Using an appropriate tool (e.g., auction site, database, service bureau) she decides to purchase a link from Rome to Paris offered by ISP A, and another link from Paris to Dublin offered by ISP B. However, Alice does not need the QoS pipe immediately; rather, she needs it for the time her remote presentation is scheduled, a few days later.

Payment may be effected in various ways (examples given later in the paper) depending on the policy of each ISP. Once the reservation has been booked, each ISP sends a credential to Alice authorizing her to use the required link at the desired time and date and for the appropriate time interval. The credentials are set to expire at the end of the reserved period. Again, depending on the way payment is handled and the policies of the ISPs and other involved parties, more than these two credentials may be required for access to be granted (this is explained later).

Just before the link needs to be established, Alice's QoS negotiation agent (QNA) will send a QoS request to the network elements (NEs) of the two ISPs to ensure that the appropriate resources have been allocated. Since two providers are involved, Alice's QNA will need to contact each ISP separately. Depending on the bandwidth reservation protocol used, Alice's QNA may communicate with a central entity within the ISP, or may negotiate a path through the ISP's network and then reserve the desired bandwidth with each network element separately.

For this discussion, we have limited ourselves to bandwidth reservation; additional QoS requirements (such as latency) may be specified within the same framework.

Spot Market Given an efficient purchasing mechanism, an "advance" booking such as the one mentioned earlier may be made even seconds before the channel will be used, so the term "spot market" is used to define a different payment regime that may be used to sell the unused network

\footnotetext{
${ }^{1}$ We use geographical identifi ers instead of IP addresses to simplify the example.
}

capacity. The "spot market" allows premium best-effort services to be sold. In this case, we are not making any promises regarding availability of bandwidth, but we say that by paying a small premium, packets may be treated favorably in the allocation of the remaining bandwidth (after the booked commitments are served).

\section{Architecture}

\subsection{Operation of the Spot Market}

Initially, the various bandwidth providers post their available capacities in the BAND-X clearing house. The system can accommodate one or more such clearing houses, since they function as announcement boards. Apart from that, the clearing house is not involved in the purchase of bandwidth.

The postings are of the form of credentials that describe the identity of the ISP and promise to abide by a set of QoS specifications between two points of the ISPs network. The credential may also contain the time period that the offer is valid (which may be different from the expiration of the credential), the price of the concession, and additional ISPrelated information, such as the path that should be taken between the two points. Offer credentials are signed by the ISP who issues them.

Customers contact the Clearing House to collect offers from the ISPs. For complex paths, a customer may need to collect more than one offer and use them together. In an environment with a single clearing house, the customer can issue queries to get lists of offers matching his or her requirements. If there are many clearing houses, the customer may dispatch an intelligent agent to collect the offers and come back with a recommendation that meets preassigned constraints (price, ISP reliability etc.), query each clearing house independently, or use a meta-search engine.

At the end of the search, the customer will hold one or more offer credentials that describe the desired path and QoS specs.

At this point, the customer has not actually purchased the bandwidth. In order to issue payment and reserve the bandwidth, a number of steps have to be taken. The customer (or the host at one of the end-points of the connection) contacts the first-hop network element (NE) and activates the reservation protocol. The NE issues a challenge which is then returned signed by the customer. This response also contains the offer credentials collected by the customer and a credit-worthiness credential issued by the customer's credit institution, as shown in Figure 1.

This exchange accomplishes the following: $(a)$ identifies the customer (the key that has signed the NE challenge), $(b)$ provides proof of good standing (the credential issued by 


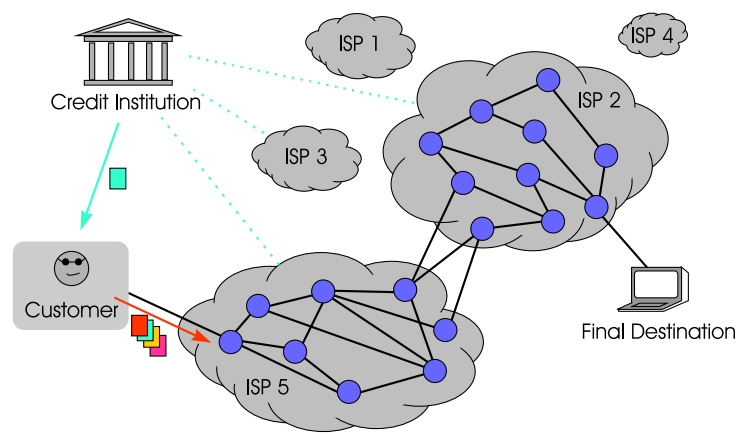

Figure 1. The customer issues a reservation request by sending the offer credentials collected from the BAND-X Clearing House along with a credit-worthiness credential issued by his or her credit institution.

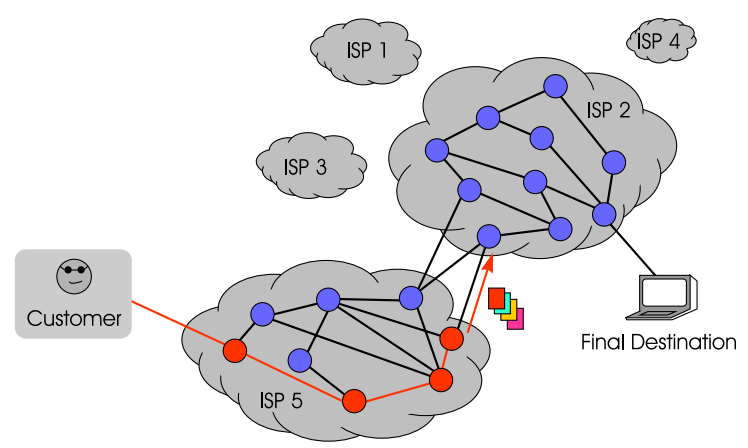

Figure 2. Each time the path crosses ISP boundaries, additional negotiations have to be carried out, to ensure that the next-hop ISP can be paid for passage.

the credit institution to the customer's key), (c) limits payment only to the offer credentials provided, $(d)$ can be used only for that particular transaction since it depends on the challenge issued by the NE. On the basis of this transaction, the first hop NE contacts other NEs within the ISPs network establishing the purchased path. If the path crosses ISP boundaries, additional transactions have to be carried out between the NE of the new ISP and the end user, as shown in Figure 2.

When the last hop is reached, the connection is considered established and the final destination host can initiate a connection with the customer's host over the reserved path (Figure 3).

There is no need for the ISPs offers to match exactly the requirements of the customer. For example, if Alice requires a 50Mbps link from Atlanta to Dublin, she may use an offer for a $100 \mathrm{Mbps}$ connection, but purchase only $50 \mathrm{Mbps}$. The providers may include clauses in their offer

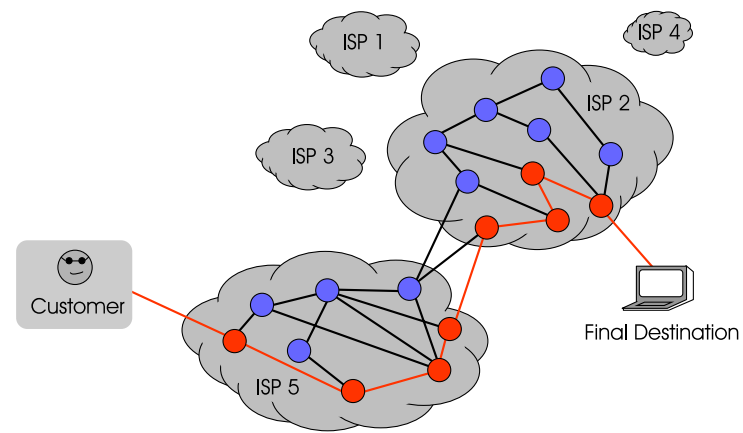

Figure 3. The path has now been established and communication can proceed.

credentials allowing or prohibiting such un-bundling. The flexibility of the policy language used in BAND-X allows many such special considerations to be encoded within the offer credentials. The advantage of having these restrictions expressed as policy is that they can be used directly by the ISP's infrastructure without any need for conversion. Moreover, the customer cannot alter these restrictions since they are an integral part of the credential (and are protected by the ISP's signing of the offer credentials).

\subsection{Operation of the Futures Market}

In the Spot Market, the customer collects the offers and sets up the path in short order, because the offers are effective immediately and have a short lifetime. There is no need to negotiate with the ISPs before the reservation.

In the Futures Market the situation is different, since the ISPs need to know what bandwidth has been purchased to plan their resource allocation. Once the customer collects the offers, a notional reservation negotiation will be initiated. The negotiation is notional because no state changes are actually effected on the network elements. The customer's QNA will not detect any change in the negotiation. Within the ISPs network, no path is created; rather the reservation is entered in the ISP's database, and a reservation credential is sent to the end user. This credential will then be used in the same manner as the offer credential was used in the Spot Market scenario. Since the bandwidth has been paid for, the reservation credential commits the ISPs to provide the requested resources at the appropriate future time.

At that time (when the path is actually required) the customer initiates a reservation negotiation, but sends only the reservation credential (instead of the offer and credit institution credentials). The ISP network elements will reserve the path as specified in the reservation credential. The case of multiple ISPs is handled in a similar manner. 


\subsection{Role of the Credit Institution}

Like the Clearing House, there is no requirement to have a single Credit Institution. It is, however, important that the ISPs have a way of confirming the keys of the various Credit Institutions. This is because the credit-worthiness credentials (CWCs) issued by the Credit Institutions to their customers will have to be verified by each ISP. If an ISP cannot verify a CWC, then it may be fake; trusting it may result in the equivalent of a bounced check.

\section{Implementation}

\subsection{KeyNote Microchecks}

The micro-payments system introduced in [3] forms the basis of our approach. The general architecture of this micro-billing system is shown in Figure 4. Under BAND$\mathrm{X}$, a Merchant is an ISP selling bandwidth and a Payer is a client wishing to make a QoS reservation.

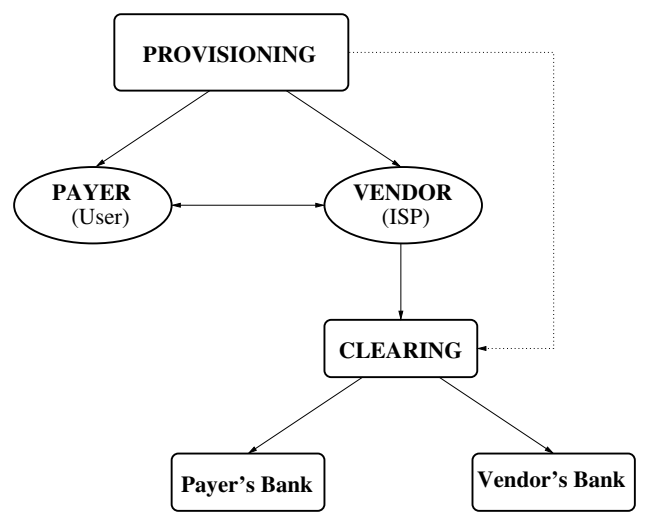

Figure 4. Microbilling architecture diagram.

In this system, Provisioning issues KeyNote [2] credentials to users (Payers) and ISPs (Merchants). These credentials describe the conditions under which a user is allowed to perform a transaction (i.e., the user's credit limit) and the fact that a Merchant is authorized to participate in a particular transaction.

Initially, the ISP encodes the details of the available bandwidth into an offer which is uploaded to the BAND$\mathrm{X}$ site, along with a credential that authorizing any user to utilize the bandwidth under the same conditions as those enclosed in the offer. Once the user finds an offer (and associated credential) that is acceptable, she must issue to the ISP a microcheck for this offer. The microchecks are encoded as KeyNote credentials that authorize payment for a specific transaction. The user creates a KeyNote credential signed with her public key and sends it, along with her credential from Provisioning, to the first network element of the ISP. This credential is effectively a check signed by the user (the Authorizer) and payable to the ISP (the Licensee). The conditions under which this check is valid match the offer sent to the user by the ISP. Part of the offer is a nonce, which maps payments to specific transactions, and prevents double-depositing of microchecks by the ISP.

To determine whether he can expect to be paid (and therefore whether to accept the payment), the ISP passes the action description (the attributes and values in the offer) and the user's key along with the ISP's policy (that identifies the Provisioning key), the user credential (signed by BAND-X ), the offer credential (signed by the ISP), and the microchecks credential (signed by the user) to his local KeyNote compliance checker. If the compliance checker authorizes the transaction, the ISP is guaranteed that Provisioning will allow payment. The correct linkage among the Merchant's policy, the Provisioning key, the user key, and the transaction details follow from KeyNote's semantics [2]. If the transaction is approved, the ISP can configure the appropriate routers such that the user's traffic is treated according to the offer, and store a copy of the microcheck along with the user credential and associated offer details for later settlement and payment.

Periodically, the ISP will 'deposit' the microchecks (and associated transaction details) he has collected to the Clearing and Settlement Center (CSC). The CSC may or may not be run by the same company as the Provisioning, but it must have the proper authorization to transmit billing and payment records to the Provisioning for the customers. The CSC receives payment records from the various ISPs; these records consist of the offer, and the KeyNote microcheck and credential from the user sent in response to the offer. In order to verify that a microcheck is good, the CSC goes through a similar procedure as the ISP did when accepting the microcheck. If the KeyNote compliance checker approves, the check is accepted. Using her public key as an index, the user's account is debited for the amount of the transaction. Similarly, the ISP's account is credited for the same amount.

\subsection{BAND-X Operation}

Having seen the overall system architecture, let us look at a particular example. Alice is a user who wants to reserve some bandwidth for a particular link with Nick's ISP. Every evening Alice contacts her banker and obtains a fresh Check Guarantor credential, which allows her to issue KeyNote microchecks. The CG credential (most of the hex digits from the keys have been removed for brevity) allows Alice to write checks for up to 5 US Dollars, and she can do so until March 24th, 2004. 


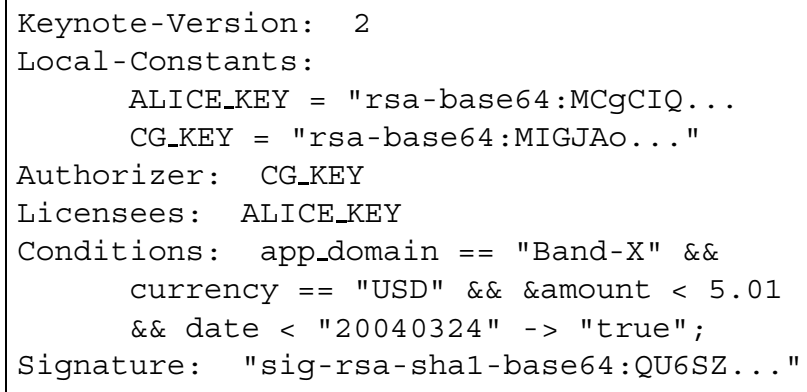

Alice now wants to reserve some bandwidth to Dublin. She searches the BAND-X for a suitable offer, and locates one issued by Nick's ISP that contains the following Offer credential, indicating that she could purchase $50 \mathrm{Mbps}$ on the specific link ("Dublin-NYC") for 3 US dollars:

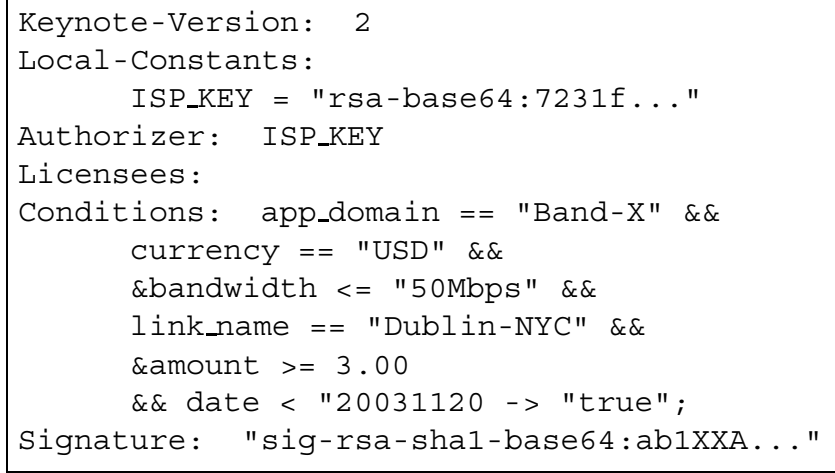

Alice then writes a check for the appropriate amount:

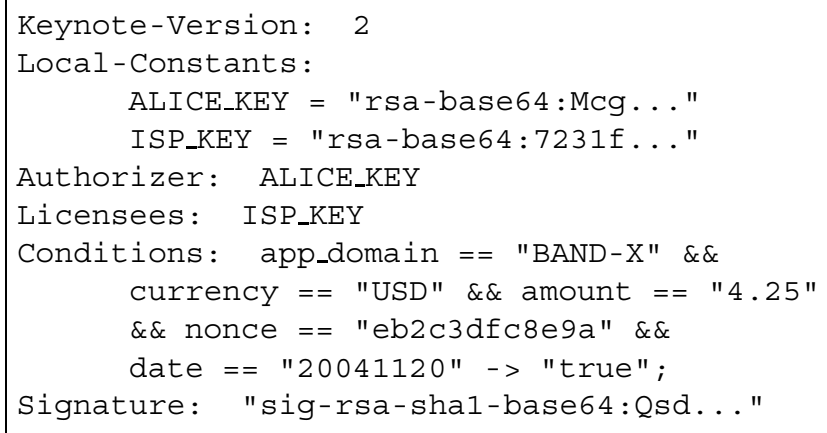

The nonce is a random number that must be different for each check, guaranteeing that there will be no doubledepositing of checks. Alice then sends the Offer credential and the micro-check to Nick's router using a protocol such as RSVP. Nick receives these credentials, validates the microcheck to make sure that he will get paid, and configures the router appropriately. If the check is not good, Nick will say so, and refuse to accept the file. Nick will verify that he will get paid, and will evaluate the Offer credential and the microcheck using a simple policy such as:

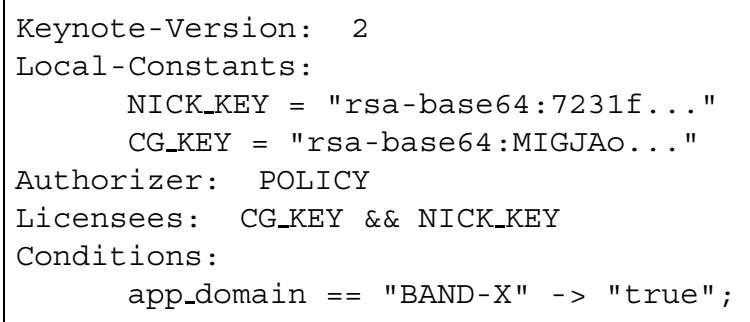

This policy says that anything that Nick's key and the Check Guarantor's key jointly authorize is allowed. Thus, Alice must submit a valid payment and a valid Offer credential. Since the bandwidth was paid for, and a path can be found from POLICY to a user (Alice) that has delegated to Nick's key, which in turn has created an open-access Offer credential, the operation is allowed. As a matter of business practice, Nick may require periodic payments from Alice in order to keep the bandwidth reserved. Alice must know that and send microchecks at the appropriate intervals.

If additional routers need to be configured in Nick's ISP, the first router forwards the necessary information to the next. Note that it is not necessary for the router itself to perform the signature verifications and policy validations: it can simply refer these operations to a Policy Decision Point (PDP), as is envisioned by the IntServ architecture.

\subsection{Security Analysis}

Similar to [3] and [8], our system has three types of communication: provisioning, reconciliation, and transaction. Although delegation of credentials (and thus access rights to reserved bandwidth) is possible, we do not consider it in this paper. We shall not worry about any value transfers to banks, as there already exist well-established systems for handling those. All communications between BAND$\mathrm{X}$, ISPs, and users can be protected with existing protocols such as IPsec or TLS. This covers both provisioning and reconciliation, which occur off-line from the actual bandwidth reservation and use. Furthermore, the transactions themselves (establishing the QoS pipes, or the right to use existing pipes) can be protected through the same means; the only requirement is that the user can authenticate with each ISP.

The confidentiality of the transmitted data itself is not within the purview of our system, nor is it a responsibility of the ISP; if the users do not trust the network with respect to data confidentiality or integrity, they should use end-to-end security protocols, e.g., IPsec or TLS. We do not impose any limitations that would preclude the use of these protocols.

The user needs to ensure that the ISPs provide the promised service. This can be easily verified by the user using a number of existing protocols and tools. Protecting against over-charging ISPs is also straightforward: the details of each transaction can be verified at any point in 
time, by verifying the credentials and the offer. Since only the user can create microchecks, a dispute claim can be resolved by "running" the transaction again. Thus, the user is safe even from a collusion between any number of ISPs and the BAND-X service. The ISP must ensure that they are paid for the services offered. Since it has a copy of all transactions (the BAND-X credential, the microcheck, and the offer), it can prove to the BAND-X, or any other party, that a transaction was in fact performed.

The BAND-X also needs to be paid for the services offered. Since the BAND-X does the clearing of the microchecks, the ISP has to provide the transaction logs to the BAND-X. The BAND-X can then verify that a transaction was done, and at what value. A collusion between the ISP and a user is somewhat self-contradicting: the user's goal is to minimize cost, while the ISP's is to maximize revenue, each at the expense of the other. The function of the BAND$\mathrm{X}$ is to verify each transaction (perhaps sampling, for very large numbers of transactions), debit the ISP and credit the user (presumably keeping some commission or small fee in the process): if the ISP does not give any credentials to the BAND-X, then no work was done as far as the BAND-X is concerned (and no payments are made, which benefits the user); claiming more transactions than really happened is not in the best interest of the user (so no collaboration could be expected in the direction), and the ISP cannot "fabricate" transactions. Since value is not stored in either the ISP or the user, only a reliable log of the transactions is needed at the ISP (and, optionally, at the user).

\section{Summary and Concluding Remarks}

To minimize network congestion which can cause complaints and dissatisfaction among users, ISPs overprovision their networks [6]. Unfortunately, unused bandwidth is wasted since it cannot be saved for later use. While bandwidth remains cheap, the ISPs can continue to add capacity ahead of the actual demand, but this state of affairs will only last as long as users of time-sensitive services prefer the telephony network. The enormous cost difference between the telephony network and the Internet provides an implicit subsidy. However, as users switch to the Internet for their time-sensitive services, ISPs will no longer be able to expand their networks. We believe that the framework described in this paper offers a migration path for both users and ISPs through the creation of an open market for bandwidth over the Internet. The reason is that the BAND$\mathrm{X}$ framework supports a competitive market offering transparency, and security. At the same time the low overheads of the BAND-X framework ensure scalability through the use of a micro-payment environment.

The benefits offered by BAND-X include: $(a)$ "instant" purchases of bandwidth and advanced purchases allowing the ISPs to plan ahead their resource allocation strategies, while being able to auction off unused capacity rather that letting it go at Best-Effort prices, $(b)$ efficiency, requiring only a few exchanges between a buyer and sellers to effect a reservation. Moreover, the use of the Keynote-based micro-payment framework provides system-wide efficiency and scalability, $(c)$ compatibility with existing standards: by utilizing an existing reservation protocol (RSVP), a BAND$\mathrm{X}$ system may be be deployed with minimum disruption. (d) trades between parties that have no established business relationships: The Credit Institution(s) link buyers and sellers, thus allowing a transaction to go through without the need for a buyer to be known to the seller. This is a key requirement for the bandwidth market to work freely with the buyer being able to select the seller offering the best value for money. (e) openness: the BAND-X model allows the presence of multiple entities for each role (i.e., we can have multiple Credit Institutions, Clearing Houses, buyers and sellers) operating within a single market. This increases the competition and overall reliability of the entire system.

\section{References}

[1] S. Blake, D. Black, M. Carlson, E. Davies, Z. Wang, and W. Weiss. An Architecture for Differentiated Services. Technical report, IETF RFC 2475, December 1998.

[2] M. Blaze, J. Feigenbaum, J. Ioannidis, and A. D. Keromytis. The KeyNote Trust Management System Version 2. Internet RFC 2704, September 1999.

[3] M. Blaze, J. Ioannidis, and A. D. Keromytis. Offine Micropayments without Trusted Hardware. In Proceedings of the Fifth International Conference on Financial Cryptography, 2001.

[4] R. Braden, L. Zhang, S. Berson, S. Herzog, and S. Jamin. Resource ReSerVation Protocol (RSVP) - Version 1 Functional Specifi cation. Internet RFC 2208, 1997.

[5] L. Burgstahler, K. Dolzer, C. Hauser, J. Jähnert, S. Junghans, C. Macián, and W. Payer. Beyond Technology: The Missing Pieces for QoS Success. In Proceedings of the ACM SIGCOMM Workshop on Revisiting IP QoS (RIPQOS), held in conjunction with the ACM SIGCOMM conference, August 2003.

[6] M. Currence, A. Kurzon, D. Smud, and L. Trias. A Causal Analysis of Usage-Based Billing on IP Networks, 2000.

[7] B. Davie. Deployment Experience with Differentiated Services. In Proceedings of the ACM SIGCOMM Workshop on Revisiting IP QoS (RIPQOS), held in conjunction with the ACM SIGCOMM conference, August 2003.

[8] J. Ioannidis, S. Ioannidis, A. Keromytis, and V. Prevelakis. Fileteller: Paying and Getting Paid for File Storage. In Proceedings of the Sixth International Conference on Financial Cryptography, March 2002. 